Bryn Mawr College

Scholarship, Research, and Creative Work at Bryn Mawr

College

Physics Faculty Research and Scholarship

Physics

1995

\title{
Young's Double-Slit Interferometry within an Atom
}

Michael W. Noel

Bryn Mawr College, mnoel@brynmawr.edu

C. R. Stroud Jr.

Let us know how access to this document benefits you.

Follow this and additional works at: http://repository.brynmawr.edu/physics_pubs

Part of the Physics Commons

\section{Custom Citation}

Michael W. Noel and C. R. Stroud, Jr., "Young's double-slit interferometry within an atom," Phys. Rev. Lett. 75, 1252 (1995).

This paper is posted at Scholarship, Research, and Creative Work at Bryn Mawr College. http://repository.brynmawr.edu/physics_pubs/78

For more information, please contact repository@brynmawr.edu. 


\title{
Young's Double-Slit Interferometry within an Atom
}

\author{
Michael W. Noel and C. R. Stroud, Jr. \\ The Institute of Optics, University of Rochester, Rochester, New York 14627 \\ (Received 10 April 1995)
}

\begin{abstract}
An experiment is described which is an analog of Young's double-slit interferometer using an atomic electron instead of light. Two phase-coherent laser pulses are used to excite a single electron into a state of the form of a pair of Rydberg wave packets that are initially on opposite sides of the orbit. The two wave packets propagate and spread until they completely overlap, then a third phase-coherent laser pulse probes the resulting fringe pattern. The relative phase of the two wave packets is varied so that the interference produces a single localized electron wave packet on one side of the orbit or the other.
\end{abstract}

PACS numbers: 03.75.Dg, 32.80.Rm, 42.25.Hz

Young's double-slit interference is fundamental to our understanding of the coherence properties of any wave phenomenon. It has played a major role in the development of optical coherence theory [1]. Double-slit interference has also been observed with the de Broglie waves of free electrons [2,3]. In this paper we describe an experiment in which Rydberg wave packets are used to study Young's double-slit interference within an atom. There have been a number of experiments in recent years in which a picosecond laser pulse is used to excite an electron into a linear combination of highly excited states in the form of a spatially localized wave packet traveling around a classical Kepler orbit [4,5]. Here we split one electron into two separate wave packets and inject the two packets into the opposite sides of a Rydberg-Kepler orbit. This is analogous to a Young's double-slit experiment with the two slits on the opposite sides of the orbit, a distance of approximately one-half of a micron. By using phase-locked laser pulses to excite the two wave packets we can control the relative phase of the de Broglie wave of the two packets. After the two packets have traveled a number of times around the Kepler orbit, and each has spread, the interference is quite dramatic with the relative phase actually able to determine on which side of the orbit a well-localized single-wave-packet revival occurs.

Although the analogy between our wave packet interferometer and Young's interferometer is not exact, it provides an intuition useful for interpreting our experiment [6]. In Young's interferometer light is passed through an opaque screen containing two slits. The two slits form secondary light sources. The light from these secondary sources is then allowed to propagate, and interference is viewed on a distant screen. The best fringe visibility is seen in the region where the light from the two sources exactly overlaps spatially. The interferometer in our atom is somewhat different. The wave packets are created by two temporally separated laser pulses. The result is the excitation of two spatially separated radial electron wave packets which move around a common Kepler orbit. It is the evolution of these two wave packets in the atomic potential that eventually allows them to spread around the entire orbit so they spatially overlap and interfere.
As a radial wave packet propagates around its orbit it spreads due to the anharmonic nature of the atomic potential. The dynamics of the wave packet in this anharmonic potential have been studied in detail by several authors [7-12] (for an early review see Ref. [13]). They find that the wave packet does not remain dispersed, but eventually becomes relocalized, since it is a superposition of discrete eigenstates. The time for the wave packet to relocalize is called the revival time $T_{R}$. At small integer ratios of the revival time the wave packet can be well localized at more than one radial position. It is during these "fractional revivals" that the initially excited wave packet pair can be maximally overlapped, and the best interference fringe visibility can be seen.

The delay between the two laser pulses used to excite the radial wave packets is precisely controlled to excite a state that looks like a one-half fractional revival. The onehalf fractional revival is the form that a single-electron wave packet takes when it has spread all of the way around the orbit and interference between the head and tail of the packet produces a fringe pattern in the form of two miniature replicas of the original wave packet located on opposites sides of the orbit $[7,9,11,12]$. The phase relation between the pair of wave packets in the initial fractional-revival-like state can be chosen arbitrarily. This is in contrast with the fractional revival resulting from the evolution of a wave packet excited by a single pulse which has a specific phase between subwave packets. By controlling this phase we can manipulate the interference between the two wave packets, which occurs at a later time. A third laser pulse is used to probe the evolution of this superposition state and study the interference between the two excited wave packets.

The use of phase coherent pulses to study radial wave packets was first proposed a few years ago and has since been used in several experiments [14-17]. In these studies a single pump pulse is used. The pump pulse excites a radial wave packet. This wave packet is allowed to evolve for a given time. Next, a second identical laser pulse is sent in to probe the wave packet evolution. If the original wave packet is near the core it can interact strongly with the probe pulse. The result of 
this interaction depends on the phase relationship between the wave packet and the probe pulse as in a Ramsey fringe experiment [18]. If the two are in phase more population is coherently pumped into the excited state. If the two are out of phase, the excited wave packet is stimulated to make a transition back to the ground state leaving no population in the excited state. When the wave packet is not near the core the probe pulse does not interact with the excited wave packet. By measuring the excited state population after the probe-pulse interaction we can determine the location of the wave packet. If large oscillations at the optical period are seen in the population (Ramsey fringes) then the wave packet was near the core at the time of the probe pulse. No oscillation at the optical period indicates the wave packet was not near the core during the probe's interaction. This technique allows for an excellent signal-to-noise ratio due to the efficiency with which the excited state population can be measured using simple dc field ionization.

In our experiment we use a $Q$-switched mode-locked Nd:YAG laser to synchronously pump a dye laser. The dye laser is cavity dumped at the $Q$-switch repetition rate of $800 \mathrm{~Hz}$ to produce a $10 \mu \mathrm{J}$ laser pulse of 25 ps duration. After frequency doubling, the pulse is sent into a series of beam splitters, delay lines, and retroreflecting mirrors that produce a train of three pulses (Fig. 1). Each is identical to the first pulse, but with relative delays between the pulses that are controlled to approximately one-hundredth of an optical period. To achieve this degree of control, an active

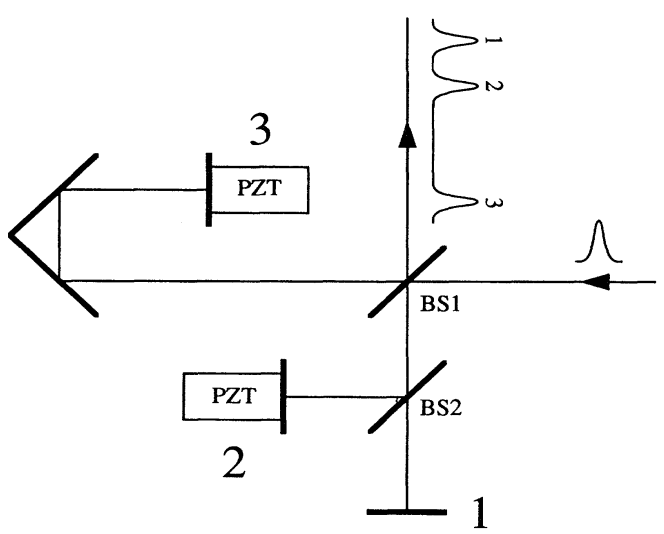

FIG. 1. Apparatus used to create a phase-coherent three-pulse sequence. A single pulse from the dye laser enters from the right of the figure, and the three-pulse train exits to the top of the figure toward the atomic beam. The path length difference between arms 1 and 2, and between arms 1 and 3 are independently interferometrically stabilized. This is done by sending a reference beam from a $\mathrm{HeNe}$ laser into the interferometer formed by arms 1 and 2. A piece of glass with a small wedge is placed in the $\mathrm{HeNe}$ path in arm 1 to produce tilt fringes at the output. A fringe stabilization circuit produces an error signal from these tilt fringes which is fed back to the piezoelectrically driven mirror in arm 2. An independent $\mathrm{HeNe}$ beam and fringe stabilizer are used to control the path length difference between arms 1 and 3 . servo stabilization system is used with a HeNe reference laser. The three-pulse train is focused onto a potassium atomic beam where the wave packets are excited. After this interaction the excited state population is measured by applying a dc electric field pulse, which ionizes all of the population from the Rydberg series. The ions are collected with an electron multiplier and counted for the various configurations described below.

The experiment illustrated in Fig. 1 proceeds in two parts. In the first part the motion of a single radial wave packet is measured to serve as a calibration run. To do this, the second pump pulse (label 2 in the diagram) is blocked and the signal from the radial electron wave packet excited by pulse 1 is measured with the phase sensitive pumpprobe technique. In our implementation of this technique the delay between pulses 1 and 3 is scanned in two ways. A corner cube mounted on a translation stage is used to take coarse delay steps. At each of these coarse delays the servo system is turned on and fine delay steps are taken with the mirror mounted on the piezoelectric cylinder. This is done by translating the detector, which is used in the fringe stabilization circuit across the fringe pattern causing the circuit to pull the fringes with it by moving the mirror in arm 3. So, the raw data consist of a few Ramsey fringes at each of the discrete coarse delay steps. The amplitude of these fringes is a measure of where the radial wave packet is in its evolution. Figure 2 shows the evolution of the
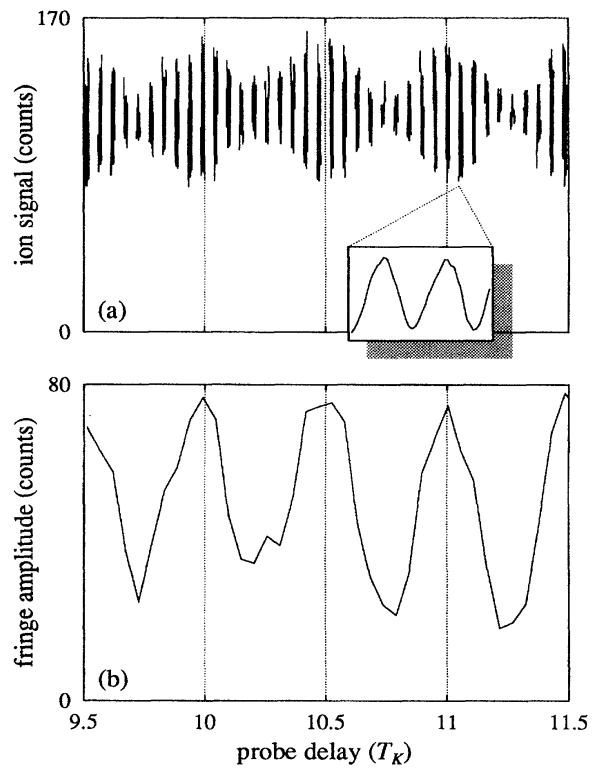

FIG. 2. Radial wave packet evolution for excitation with a single pump pulse. The laser frequency is tuned to excite a wave packet near $\bar{n}=66$ in potassium. The Kepler period of the orbit is $T_{K}=44 \mathrm{ps}$. The Ramsey fringe signal is shown in (a) with a single fringe in the inset. The amplitude of each of the Ramsey fringes is plotted in (b). At this delay time near the one-half fractional revival $\left(\frac{1}{2} T_{R}=11 T_{K}\right)$ the wave packet is split into two pieces which pass the core at integer and halfinteger multiples of the Kepler period. 
wave packet near the one-half fractional revival. There are peaks separated by half the Kepler period indicating that the wave packet is localized at two spatial positions.

In the second part of the experiment we unblock the second pump pulse and look at the interference between two wave packets. (A similar type of wave packet interference was considered by Alber, Ritsch, and Zoller [8]. An interferometer within a molecule was described by Garraway and Stenholm [19].) The delay between the first and second pump pulses is set to one-half of the Kepler period so that a state like the one-half fractional revival is excited. The interference between these two wave packets is investigated at a time near one-half of the revival time. The reason for this choice of probe time becomes obvious if we look at each wave packet individually (Fig. 3). The first wave packet will have evolved to the one-half fractional revival. The two subwave packets of this state will pass the core at integer and half-integer multiples of the Kepler period as was described above. The wave packet produced by the second pump pulse will also have evolved to the onehalf fractional revival state. Since the delay between the two pump pulses was chosen to be one-half of the Kepler

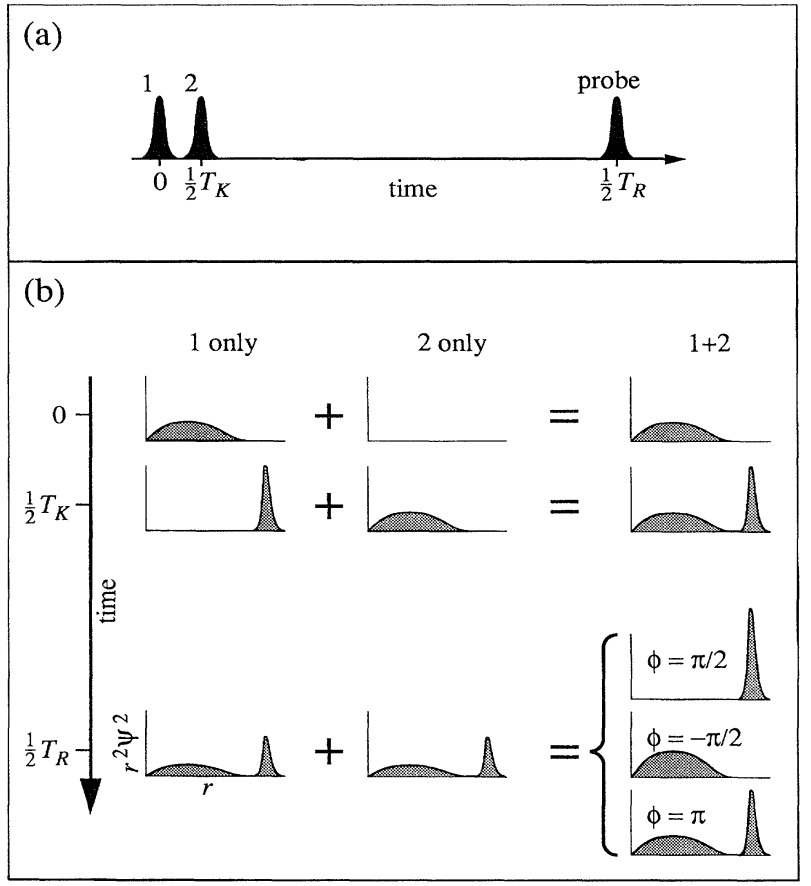

FIG. 3. (a) Time line for the laser pulse interaction. (b) Cartoon plots of the radial probability distribution $r^{2} \psi^{2}$ of the electron as a function of radius $r$. The first column ( 1 only) shows the evolution of the wave packet excited by pulse 1 as if pulse 2 were not there. Column two shows the wave packet evolution resulting from pulse 2 only. The third column $(1+2)$ shows the evolution resulting from the coherent sum of the two wave packets. Near the half revival the localization resulting from the coherent sum varies depending on the exact phase relation between the wave packets. The resulting localization for three particular choices of phase is shown. period, each of the initially excited wave packets will have evolved into states which exactly spatially overlap as they pass the core near the one-half revival time. So, at this probe time the wave packets excited by the individual pump pulses can strongly interfere.

Figure 4 shows the evolution of a state excited with the pair of phase coherent laser pulses for three particular choices of phase difference. In the first case [Fig. 4(a)] the phase was chosen so that the wave packets from the two pump pulses which pass the core at integer multiples of the Kepler period exactly cancel, and the packets which pass the core at half-integer multiples of the Kepler period add constructively. The result is a single welllocalized wave packet oscillating at the classical period: a full revival. This phase was found experimentally by fixing the probe delay at $10 T_{K}$ and monitoring the Ramsey fringe amplitude as the phase relationship between the two pump pulses was varied. Once the fringe amplitude was minimized, the phase between pump pulses was locked and kept fixed as the delay of the probe pulse was scanned to look at the wave packet evolution.
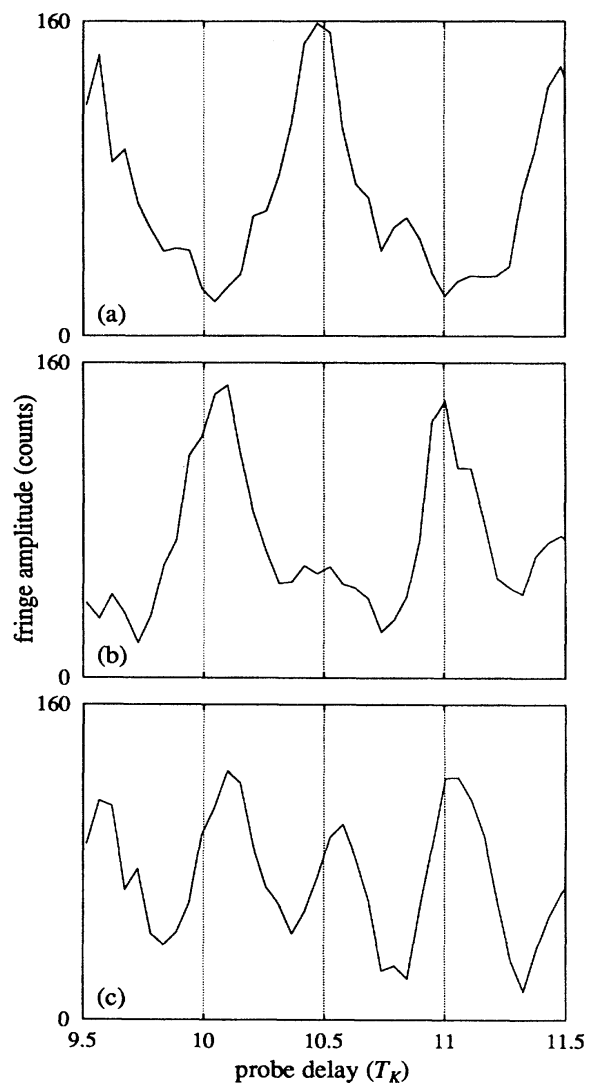

FIG. 4. Radial wave packet evolution for excitation with a pair of phase coherent pump pulses. Probe delay is measured from the first pump pulse and is in units of the Kepler period. The phase between pump pulses modifies the interference seen between the two wave packets near one-half of the revival time. The phases are approximately (a) $\pi / 2$, (b) $-\pi / 2$, and (c) $\pi$. 
The next choice of phase [Fig. 4(b)] has a very similar effect. Again, the wave packet is localized at one spatial position at the probe time; however, now it passes the core at integer multiples of the Kepler period. This is identical to the behavior of a wave packet excited with a single laser pulse and probed at a time near $2 T_{R}$. Therefore the pump pulse pair has excited the $3 / 2$ fractional revival.

For the choice of phase in Fig. 4(c) the wave packet is still split into two subpackets at the probe time. In fact, for this case the wave packet will never come to a full revival. Hence, this wave packet has no analog in a wave packet excited by a single laser pulse.

The examples in Fig. 4 show the dramatic effect that a simple change in phase can have on the future evolution of a wave packet excited with this interferometric technique. For two special choices of phase the $1 / 2$ and $3 / 2$ fractional revivals can be excited as shown in (a) and (b), respectively. The phases for these two special cases differ by $\pi$. This technique also allows for the excitation of a fractional revival state with arbitrary phase between subwave packets as in (c). It can be generalized further by changing the coarse delay between pump pulses, looking at other probe times, investigating higher order fractional revivals, or interfering wave packets of two colors. These examples illustrate via a generalization of the Young's double-slit experiment that the coherence properties of atomic electron de Broglie waves dramatically affect Rydberg wave packet evolution, and that by control of the phase of these waves we can reproduce the interference effects of classical optics.

We would like to acknowledge helpful discussions with J. Bromage, Z. Gaeta, and M. Mallalieu. This work was supported in part by the Army Research Office.

[1] Max Born and Emil Wolf, Principles of Optics (Pergamon, Oxford, 1980), p. 260.
[2] C. Jönsson, Z. Phys. 161, 454 (1961).

[3] C. Jönsson, Am. J. Phys. 42, 4 (1974). Translation by D. Brandt and S. Hirschi.

[4] A. ten Wolde, L. D. Noordam, A. Lagendijk, and H. B. van Linden van den Heuvell, Phys. Rev. Lett. 61, 2099 (1988).

[5] John A. Yeazell, Mark Mallalieu, Jonathan Parker, and C. R. Stroud, Jr., Phys. Rev. A 40, 5040 (1989).

[6] Various aspects of our experiment have analogies with different types of interferometers. In particular, the evolution of a single wave packet can be compared to the self-imaging properties of a Talbot interferometer. For a nice discussion of these interferometers, see K. Patorski, in Progress in Optics, edited by E. Wolf (North-Holland, Amsterdam, 1989), Vol. 27.

[7] Jonathan Parker and C. R. Stroud, Jr., Phys. Rev. Lett. 56, 716 (1986).

[8] G. Alber, H. Ritsch, and P. Zoller, Phys. Rev. A 34, 1058 (1986).

[9] I. Sh. Averbukh and N. F. Perelman, Phys. Lett. 139A, 449 (1989).

[10] John A. Yeazell, Mark Mallalieu, and C. R. Stroud, Jr., Phys. Rev. Lett. 64, 2007 (1990).

[11] John A. Yeazell and C. R. Stroud, Jr., Phys. Rev. A 43, 5153 (1991).

[12] D. R. Meacher, P.E. Meyler, I. G. Hughes, and P. Ewart, J. Phys. B 24, L63 (1991).

[13] G. Alber and P. Zoller, Phys. Rep. 199, 231 (1991).

[14] L. D. Noordam, D. I. Duncan, and T.F. Gallagher, Phys. Rev. A 45, 4734 (1992).

[15] J. F. Christian, B. Broers, J. H. Hoogenraad, W. J. van der Zande, and L. D. Noordam, Opt. Commun. 103, 79 (1993).

[16] J. Wals, H. H. Fielding, J. F. Christian, L. C. Snoek, W. J. van der Zande, and H.B. van Linden van den Heuvell, Phys. Rev. Lett. 72, 3783 (1994).

[17] R. R. Jones, C.S. Raman, D. W. Schumacher, and P. H. Bucksbaum, Phys. Rev. Lett. 71, 2575 (1993).

[18] Norman F. Ramsey, Phys. Rev. 78, 695 (1950).

[19] B. M. Garraway and S. Stenholm, Phys. Rev. A 46, 1413 (1992). 